Chirurg 2016 $\cdot 87: 907-908$

DOI 10.1007/s00104-016-0293-z

Online publiziert: 27. September 2016

(c) Springer-Verlag Berlin Heidelberg 2016

CrossMark
M. Anthuber

Klinik für Allgemein-, Viszeral- und Transplantationschirurgie, Klinikum Augsburg, Augsburg, Deutschland

\title{
Klammernahtoperationen am Anorektum
}

\section{Indikationen, Techniken, Ergebnisse}

Die Einführung von Klammernahttechniken vor mehr als 30 Jahren hat, und das kann man ohne Übertreibung feststellen, die Viszeralchirurgie revolutioniert. Kontinuitätswiederherstellungen in Handnahttechnik am oberen und unteren Gastrointestinaltrakt waren aufgrund der beengten und tiefen Verhältnisse im operativen Situs überaus zeitaufwendig, technisch höchst anspruchsvoll und nicht zuletzt deshalb anfällig hinsichtlich der Entwicklung von Nahtinsuffizienzen. Die Entwicklung linearer und zirkulärer Klammernahtgeräte hat einen wichtigen Beitrag zu der Senkung lebensbedrohlicher Komplikationen ausgehend von Anastomoseninsuffizienzen geleistet, wenngleich deren Auftreten nicht vollkommen eliminiert ist. Vorrausetzung für die Anwendung moderner Stapler sind fundierte Kenntnisse über deren Handhabung und mögliche (jedoch außerordentlich seltene) geräteassoziierte Fehlermöglichkeiten und schließlich bei welcher Indikation der Einsatz sinnvoll und vor allem für den Patienten gewinnbringend ist.

Vor mehr als 25 Jahren wurde abseits der bis dahin üblichen Indikationen bei Magen-, Kolon- und Rektumresektionen die höhergradige Hämorrhoidenerkrankung als neues Anwendungsgebiet für den Zirkularstapler identifiziert. Antonio Longo hat mit seinen Vorstellungen zur normalen Anatomie des Analkanals und zur Pathophysiologie der Entstehung von Hämorrhoiden die bisher bekannten offenen Operationsverfahren (bei allen im Wesentlichen eine Resektion der prolabierenden Hämorrhoidalpolster) herausgefordert. Sein therapeutischer Ansatz umfasste zwei Komponenten: die dau- erhafte Reposition des intermittierenden partiellen Analprolapses und die gleichzeitige Drosselung der Blutzufuhr zu den Hämorrhoiden. Seine Operationstechnik fand unter dem Begriff „Anopexie“ Eingang in die medizinische Literatur. Die Durchführung erfolgte mit einem modifizierten, transanal eingeführten Zirkularstapler. Die Ergebnisse vor allem im Hinblick auf den postoperativen Schmerz waren so überzeugend, dass eine Vielzahl von Studien durchgeführt wurde, welche die Resultate von Antonio Longo weitestgehend bestätigten. Es kam zu einer raschen Verbreitung dieser Technik, die höhere operative Kosten verursacht und auf den ersten Blick simpel erscheint. Aber es wurden auch ernste Komplikationen (unerträgliche postoperative Schmerzen, massive Nachblutungen, Stenosen etc.) bis hin zu Todesfällen als Folge einer pelvinen Sepsis beobachtet, die in diesem Maße von den bis dahin durchgeführten offenen Hämorrhoidenoperationen nicht bekannt waren. Dies mag als Hinweis dafür gelten, dass nur die korrekte Indikationsstellung und die technisch exakte Durchführung unter Kenntnis der Fehlermöglichkeiten Grundlage guter Ergebnisse sein kann.

Vor allem ausgehend von Antonio Longo selbst und anderen italienischen Proktologen wurden weitere proktologische Erkrankungen definiert, denen als Symptom im Wesentlichen die gestörte Stuhlentleerung („obstructive defecation syndrome“, ODS) gemeinsam ist, die in Anlehnung an die Operationstechnik der Anopexie in modifizierter Technik und unter Verwendung anderer Stapler transanal angegangen wurden. Genannt seien hier in erster Linie die Rektozele und die
Intussuszeption bis hin zum kompletten externen Rektumprolaps. Die sog. STARR-Operation und nur kurze Zeit später die Contour-Transtar-Operation fanden schnell begeisterte Anhänger, wenngleich eine nicht unerhebliche Zahl erfahrener Proktologen gerade in Deutschland diesen Neuentwicklungen sehr skeptisch gegenüberstand und zur Vorsicht mahnte. Schnell wurde klar, dass diese Operationen eben nicht simpel, sondern technisch außerordentlich anspruchsvoll sind und ein ganz eigenes Spektrum an Komplikationsmöglichkeiten haben. Um diesen neuen Techniken $\mathrm{zu}$ einer schnelleren und sicheren Verbreitung $\mathrm{zu}$ verhelfen, wurden von der Herstellern der Klammernahtgeräte externe Hospitations- und interne Unterstützungskurse („proctoring“, ein in diesem Zusammenhang selten gut passender Begriff!) angeboten, um proktologisch interessierte Chirurgen mit der erforderlichen Expertise auszustatten. Mancherorts wurden jedoch basierend auf den Erfahrungen mit der Anopexie diese neue Operationsverfahren relativ unkritisch aufgegriffen und nicht immer zum Vorteil des Patienten angewendet.

Das Leitthema dieses Heftes „Klammernahtoperationen am Anorektum“ hat sich zum Ziel gesetzt, die bisherigen Entwicklungen aufzuzeigen, die Indikationen herauszuarbeiten, die technische Durchführung darzustellen und schließlich auch die aus der internationalen Literatur bekannten Ergebnisse zu präsentieren. Vier namhafte Kollegen, Oliver Schwandner aus Regensburg, Reinhard Ruppert aus München, Christoph Isbert aus Hamburg und Roland Scherer aus Berlin, die allesamt hoch anerkannte 
Proktologen und Meinungsführer in Deutschland sind, haben sich bereit erklärt, in dieser Ausgabe von Der Chirurg diese Thematik umfassend aufzuarbeiten. Dabei ist es durchaus erwünscht, dass es in den Einzelbeiträgen gewisse Überschneidungen und Redundanzen gibt, weil dadurch der Blickwinkel jedes einzelnen in diesem noch nicht wirklich gut ausgeleuchteten Gebiet besser erkennbar wird, und dem interessierten Leser in der eigenen kritischen Einschätzung mehr Spielraum lässt. Ich bedanke mich ausdrücklich bei den vier genannten Kollegen für ihr Engagement und die exzellente Kooperation bei der Gestaltung des Leitthemas. Ich hoffe, dass diese Ausgabe dazu beiträgt das Wissen um die Möglichkeiten und Grenzen dieser innovativen Operationstechnik auf breiter Front zu mehren.

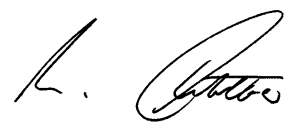

Prof. Dr. Matthias Anthuber

\section{Korrespondenzadresse}

\section{Prof. Dr. M. Anthuber}

Klinik für Allgemein-, Viszeral- und Transplantationschirurgie, Klinikum Augsburg Stenglinstr. 2, 86156 Augsburg, Deutschland Matthias.Anthuber@klinikum-augsburg.de

Interessenkonflikt. M. Anthuber gibt an, dass kein Interessenkonflikt besteht.

\section{Kiefer, T. (Hrsg.) Thoraxdrainagen}

Berlin Heidelberg: Springer-Verlag Berlin Heidelberg 2016, 1., 126 S., (ISBN: 978-3662-49739-5), Softcover 39,99 EUR

Es ist sehr schwer, innerhalb der Welt der thoraxchirurgischen Bücher ein neues Werk zu schaffen, das einzigartig ist. Das Buch Thoraxdrainagen jedoch, herausgegeben von Thomas Kiefer, erfüllt ganz klar diese Definition. Und dies gleich aus mehreren Gründen.

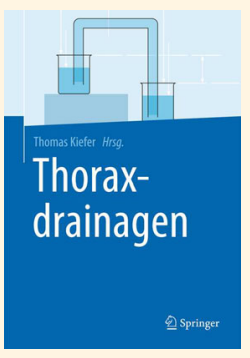

Zu allererst ist dies ein enorm praxisorientiertes Buch. Die Drainage des Pleuraspaltes und das Management von Thoraxdrainagen sind fundamentaler Bestandteil der tho-

raxchirurgischen Arbeit. Die Tatsache, dass diese Themen in dem Buch mit großer Ernsthaftigkeit besprochen werden, zeugen von seiner Intention: es will praxisorientiert sein. Das Buch will in einer einfachen, praktischen Weise hilfreich sein. Einzelne einfache Details, die bei der täglichen Arbeit helfen, die für sich genommen jedoch nicht die große Aufmerksamkeit genießen, können in ihrer Addition einen signifikanten Einfluss auf das Gelingen haben. Dieses Buch ist einzigartig in der einfachen, ehrlichen und praktischen Herangehensweise an das Thema "Thoraxdrainagen".

Das Buch stellt das Thema umfassend dar. Es umfasst die Themenfelder Anatomie, Physiologie, Indikationen, Art der Drainagen, Legen einer Drainage, Komplikationen, Pflege, Management des Pleuraspaltes, Schmerztherapie, Physiotherapie und Entfernen der Drainage. Ich habe viel gelernt, und ich wette, dass jeder Thoraxchirurg, egal wie erfahren er sein mag, in diesem Buch viele Dinge finden wird, die interessant, überraschend und hilfreich sind und die ihm helfen, seine Arbeit für und mit den Patienten zu verbessern. Einzigartig ist das Buch auch darin, sich durchgängig auf das zu beziehen, was wir aufgrund wissenschaftlicher Arbeit wissen. Ganz besonders im Umgang mit Thoraxdrainagen ist die Vorgehensweise der Kliniker häufig geprägt von Gewohnheiten und Traditionen, die vorhandene wissenschaftliche Evidenz tritt zumeist in der Hintergrund.
Dieser Bereich illustriert sehr gut die Beobachtung von Stuart Chase, die er bereits 1888 gemacht hat: „Für die, die glauben, braucht es keinen Beweis, für die, die nicht glauben, ist kein Beweis möglich". Trotz der dogmatischen Glaubensgrundsätze und Lager, die existieren, gibt es eine große Zahl seriöser Daten. Einige der wissenschaftlichen Themen sind sicherlich noch nicht umfassend aufgearbeitet, aber Thomas Kiefer hat die existierenden wissenschaftlichen Erkenntnisse gründlich aufgearbeitet. Er hat diese Erkenntnisse an jeder Stelle in einer gut lesbaren, einfachen Art und Weise eingebracht, immer offen und ehrlich betonend, was wir wissen und was wir nicht wissen. Dies führt dazu, dass er einige der häufig zitierten Dogmen entlarvt - er tut dies in einer einfachen, an Fakten orientieren und sachlichen Art und Weise. Dieser aufrichtige und sachliche Stil der Diskussion und das sich daraus ergebende Management, basierend auf den vorliegenden wissenschaftlichen Erkenntnissen, zeichnen dieses Buch im Besonderen aus.

Schlussendlich ist dieses Buch geschrieben, um im klinischen Alltag zu unterstützen und zu helfen. Es enthält Aspekte der ärztlichen wie der pflegerischen und der physiotherapeutischen Arbeit, was sich auch in der Auswahl der Co-Autoren widerspiegelt. Der Gesamteinfluss des Buches wird größer sein als seine einzelnen Kapitel. Obwohl das Buch ein alltägliches Thema zu behandeln scheint, empfehle ich es jedem, der sich mit Thoraxdrainagen in seiner klinischen Tätigkeit beschäftigt, der seine Arbeit für den Patienten durch Fokussierung auf die einfachen, täglichen Routineabläufe erheblich verbessern möchte und der sich nicht nur auf die "glamourösen" Themen konzentrieren will.

Frank C. Detterbeck MD, New Haven, CT United States 